\title{
Lack of efficacy of eplerenone for treatment of active central serous chorioretinopathy
}

\author{
Srinivas R. Sadda ${ }^{1,2}$
}

Received: 29 January 2020 / Accepted: 29 January 2020 / Published online: 10 February 2020

(c) The Royal College of Ophthalmologists 2020

Central serous chorioretinopathy (CSCR) is a common retinal disorder that if persistent, recurrent, or chronic, can lead to permanent vision loss [1]. Although there have been considerable advances in recent years in our understanding of the anatomic characteristics and imaging features of the condition, the etiology and pathophysiology of the disorder remains incompletely understood. The waxing and waning nature of CSCR and the propensity of CSCR to spontaneously improve with resolution of subretinal fluid (SRF), is an important challenge in our ability to assess the efficacy of potential therapeutic interventions. Historically, thermal laser photocoagulation was used to treat cases with persistent fluid and vision loss [2]. The justification for applying this treatment was largely based on small case series, and not randomized clinical trial data. In addition, the pathophysiologic rationale for thermal laser was uncertain. Subsequently, verteporfin photodynamic therapy (PDT) applied using half-dose or half-fluence regimens was advocated as a potential therapeutic strategy based on a few small randomized trials [3]. PDT also appears to have a physiologic rationale, as treatment was associated with a reduction in choroidal thickness. However, as PDT is invasive, has potential side effects, and is expensive and not broadly available, there has been considerable interest in developing alternative therapeutics. Recently, the use of mineralocorticoid antagonists has gained interest as a potential simple, oral therapeutic for patients with CSCR. As mineralocorticoids are known to have an impact on the choroidal vasculature, the pathophysiologic rationale for using these agents would appear to be sound. Randomized clinical trial evidence to support the use of these agents,

\section{Srinivas R. Sadda}

ssadda@doheny.org

Doheny Eye Institute, Los Angeles, CA, USA

2 Department of Ophthalmology, David Geffen School of Medicine, University of California, Los Angeles, Los Angeles, CA, USA however, has been lacking, which underscores the importance of the recent paper by Lotery et al. describing the results of the VICI trial [4].

The VICI trial is a randomized, double-blind, placebocontrolled study to evaluate the efficacy of eplerenone for the treatment of active, previously untreated CSCR for more than four months. Patients were treated with eplerenone or placebo (when fluid was present) for a period of 12 months. Although over 400 subjects were initially evaluated for eligibility, ultimately only 114 were enrolled and randomly assigned. Despite this, the study was adequately powered to identify a 5-letter ETDRS distance visual acuity difference between the groups, which the authors deemed would be of clinical significance. The study had exceptionally good retention and strong adherence to protocol, which provides a high level of confidence in the results. Overall, the VICI study observed no significant benefit of eplerenone with regards to distance visual acuity (the primary outcome measure) or with respect to a number of key secondary anatomic outcomes including subretinal fluid and retinal thickness, resolution of fluid, and time to recurrence. To provide further context for their findings, the authors also performed a meta-analysis of other eplerenone trials and demonstrated relatively small and presumably clinically insignificant differences between eplerenone and placebo.

The findings of the VICI trial are of substantial clinical relevance as they would not appear to support the continued use of eplerenone to treat these patients with active CSCR. Despite many strengths of this important trial, however, there are some issues, which warrant further analysis and review. First, choroidal neovascularization (CNV), particularly Type 1 or sub-retinal pigment epithelial (RPE) NV, is a known frequent complication and association of CSCR. The authors excluded CNV, but the methodology to exclude $\mathrm{CNV}$ is not specified. CNV can be difficult to identify on angiography in the setting of CSCR as it may be difficult to distinguish subtle leakage associated with Type $1 \mathrm{NV}$ from staining of CSCR-related RPE alterations or "oozing" of dye from diffusely sick RPE. NV could contribute to 
persistent SRF in eyes with CSCR and would not be expected to respond to eplerenone, whereas it may respond to PDT. It should be noted that twice as many placebotreated patients received PDT compared with the eplerenone group. Another potential concern is the very high baseline vision of the subjects enrolled in this trial (better than 6/9 in both arms). Although the trial was powered to identify a 5letter difference, it may have been difficult for any treatment to demonstrate such a margin of benefit given the starting level of vision (i.e., a problem of a "ceiling effect"). This slim chance of demonstrating a benefit of that magnitude was further diminished by the fact that even the placebo group showed an improvement of nearly four letters. In addition, though the two groups were relatively wellbalanced, there were potentially clinically relevant numerical differences in some key baseline anatomic parameters, including the SRF thickness and central subfield retinal thickness (CST), which were both greater at baseline in the eplerenone group. Furthermore, the low luminance visual acuity (LLVA) was worse at baseline in the eplerenone group. Of note, although the distance visual acuity did not reach the pre-specified 5-letter superiority margin at 12 months, the LLVA improved by 6.9 letters in the eplerenone group compared with only 1.3 letters in the placebo group-a margin of 5.6 letters.

Despite these potential concerns, the totality of the data, including in particular the 12-month change in the anatomic parameters, would appear to support the authors conclusion that eplerenone was not superior to placebo for treatment of active CSCR over a one year period. Eplerenone, however, is a relatively weak mineralocorticoid antagonist compared with spironolactone [5]. The lack of any increase in incidence of hyperkalemia in the eplerenone treated patients compared with placebo (14\% in both groups) would appear to support this contention. The authors use of eplerenone (rather than spironolactone) is certainly well-justified when considering the duration of their study and the predominance of males in the cohort, given the potential for gynecomastia associated with spironolactone. One wonders, however, whether a shorter trial using spironolactone [6], may have achieved a more substantial anatomic (and visual) response. A prospective randomized trial of spironolactone may be required in order to exclude the potential relevance of mineralocorticoid antagonism in the treatment of CSCR.

Nonetheless, the VICI investigators should be congratulated on an exceptionally well-executed study, which provides important, new information of clinical relevance which discourages the use of eplerenone for the treatment of active CSCR.

\section{Compliance with ethical standards}

Conflict of interest SRS reports honoraria from Amgen, Bayer, Genentech/Roche, Regeneron, Novartis, Allergan, 4DMT, Heidelberg, Optos, Nidek, Centervue, and Heidelberg Engineering.

Publisher's note Springer Nature remains neutral with regard to jurisdictional claims in published maps and institutional affiliations.

\section{References}

1. Mrejen S, Balaratnasingam C, Kaden TR, Bottini A, Dansingani K, Bhavsar KV, et al. Long-term visual outcomes and causes of vision loss in chronic central serous chorioretinopathy. Ophthalmology. 2019;126:576-88.

2. Slusher MM. Krypton red laser photocoagulation in selected cases of central serous chorioretinopathy. Retina. 1986;6:81-4. SpringSummer

3. Chan WM, Lai TY, Lai RY, Liu DT, Lam DS. Half-dose verteporfin photodynamic therapy for acute central serous chorioretinopathy: one-year results of a randomized controlled trial. Ophthalmology. 2008;115:1756-65.

4. Lotery A, Sivaprasad S, O'Connell A, Harris RA, Culliford L, Ellis L, VICI trial investigators. et al. Eplerenone for chronic central serous chorioretinopathy in patients with active, previously untreated disease for more than 4 months (VICI): a randomised, double-blind, placebo-controlled trial. Lancet. 2020;395:294-303.

5. Bloch MJ, Basile JN. Spironolactone is more effective than eplerenone at lowering blood pressure in patients with primary aldosteronism. J Clin Hypertens. 2011;13:629-31.

6. Lee JH, Lee SC, Kim H, Lee CS. Comparison of short-term efficacy between oral spironolactone treatment and photodynamic therapy for the treatment of nonresolving central serous chorioretinopathy. Retina. 2019;39:127-33. 